\title{
Characterizing Recovery of Sleep after Four Successive Night Shifts
}

\author{
Tomohide KUBO ${ }^{1,2 *}$, Masaya TAKAHASHI ${ }^{1}$, Norihide TACHI ${ }^{3}$, \\ Hidemaro TAKEYAMA ${ }^{4}$, Takeshi EBARA ${ }^{2}$, Tatsuki INOUE ${ }^{2,5}$, \\ Toshimasa TAKANISHI ${ }^{2,6}$, Gen-i MURASAKI ${ }^{7}$ and Toru ITANI ${ }^{8}$
}

\author{
${ }^{1}$ National Institute of Occupational Safety and Health, 6-21-1 Nagao, Tama-ku, Kawasaki 214-8585, Japan \\ ${ }^{2}$ Department of Occupational and Environmental Health, Nagoya City University Graduate School of Medical \\ Sciences, Aichi, Japan \\ ${ }^{3}$ College of Life and Health Sciences, Chubu University, Aichi, Japan \\ ${ }^{4}$ Faculty of Human Wellness, Tokaigakuen University, Aichi, Japan \\ ${ }^{5}$ Faculty of Sociology, Ryukoku University, Kyoto, Japan \\ ${ }^{6}$ Faculty of Humanities, The University of Kitakyushu, Fukuoka, Japan \\ ${ }^{7}$ Nagoya JAPAN POST Health Care Center, Aichi, Japan \\ ${ }^{8}$ Labour Protection Department, ILO, Geneva, Switzerland
}

Received April 24, 2009 and accepted July 30, 2009

\begin{abstract}
The purpose of this study was to characterize the recovery pattern of sleep following simulated, four successive night shifts in ten healthy men $(22.9 \pm 3.2 \mathrm{yr})$. Poor sleep was defined as sleep efficiency of $80 \%$ or lower as determined actigraphically. The results showed that four (rapid, slow, pseudo, and incomplete) patterns of sleep recovery were observed over three recovery sleep periods. The rapid and slow recovery pattern represented immediate and slow return to baseline level prior to the nightshifts, respectively. The pseudo recovery pattern demonstrated poor sleep at the 3rd recovery sleep period, despite transient recovery at the 2 nd sleep period. The incomplete recovery pattern was characterized by consistently poorer sleep during the entire recovery period. The correlation analysis indicated that sleep habits (bed time and variation of wake time) prior to the experiment were significantly related to the recovery patterns, rather than performance and alertness during the night shifts.
\end{abstract}

Key words: Re-adaptation to dayshift, Individual difference, Recovery cost, Actigraphy, Successive nightshifts, Recovery sleep pattern

Night shift workers suffer from sleep disturbances and chronic fatigue more often than day workers ${ }^{1)}$ due to misalignment between biological clocks and shift schedules. In particular, successive night shifts are thought to be problematic because work performance is shown to continually deteriorate over the course of successive nights ${ }^{2}$. The Shift Work Committee of the Japan Society for Occupational Health ${ }^{3)}$ recommended that night shifts should consist of up to at least two or three successive nights in conformity with the guideline put forth by Knauth and Rutenfranz ${ }^{4)}$. Some researchers, however,

*To whom correspondence should be addressed.

E-mail: kubo@h.jniosh.go.jp have proposed that performance and alertness improve over a period of successive night shifts ${ }^{5,6}$.

Although several possibilities may exist in explaining why the concept of shift schedule design is still controversial, the effect of individual differences in adaptation to night shifts may be one of the relevant factors. Individual variability in response to sleep deprivation has been mostly overlooked in the scientific literature. However, this issue has been extensively studied in recent research ${ }^{7,8)}$. Van Dongen et al. (2004) revealed that neurobehavioral performance impairment by sleep deprivation showed trait-like differential vulnerability, which is stable within individuals ${ }^{7)}$. Additionally, clock gene research suggested that PERIOD3 polymorphism was 
associated with nighttime decrements in performance and alertness ${ }^{8}$. A better understanding of individual differences in response to sleep deprivation would enable night shifts to be more effectively managed, and allow preventive strategies for human errors and shift work-related diseases to be tailored to the individual.

In terms of quality of life, however, particular attention should be paid to re-adaptation to the diurnal type of life and recovery from sleep deprivation. Many shift workers return to the diurnal type of life on their day shifts and days off, often experiencing difficulty in re-adaptation. Also, spending a lot of time sleeping at home for recovery may disturb work-life balance ${ }^{9}$. However, little is known about the recovery process from successive night shifts. Furthermore, to our best knowledge, any definite findings are not available regarding the recovery of sleep at an individual level.

The aim of the present study was to characterize the recovery process of sleep following four successive simulated night shifts. Our primary interest was to determine how the recovery pattern of sleep would be different among individuals, and subsequently to examine contributing factors to the different patterns observed.

Participants were recruited from universities in Aichi and Fukuoka prefectures according to the following criteria: "neither entirely morning nor evening type", as evaluated by the morningness-eveningness questionnaire $(\mathrm{MEQ})^{10)}$, non-smokers, no-sleep disorders, and not undertaking shift work or overseas travel in the past three months. Ten healthy men (mean \pm SD: $22.9 \pm 3.2 \mathrm{yr}$ old; mean MEQ score \pm SD: $52.6 \pm 6.6$; mean body mass index \pm SD: $22.1 \pm 2.5$ ) participated in this experiment. Before the experiment commenced, participants were given a detailed description of the study, and their informed consent was obtained in writing. Ethical approval for this study was obtained from the Ethics Committee of JAPAN POST. All participants were paid for participation. The experiment was conducted in a laboratory at the training institute of JAPAN POST.

For one week preceding the experiment, participants were prohibited from taking daytime naps, depriving themselves of sleep, and drinking beverages containing caffeine or alcohol. In addition, they were required to record their sleeping and waking times on a sleep log for one week preceding the experiment. During the experimental sessions, all participants spent their time in the laboratory for nine successive days. On the first day, participants were required to arrive at the laboratory by 14:00. The first day was adaptation and training, and participants were required to be in bed from 0:00 to 7:00 (Adaptation Sleep). On the second day, Baseline Sleep (0:00-7:00) and Day Shift-1 (10:00-18:00) were set.
Beginning on the third day and continuing for a total of $4 \mathrm{~d}$, participants were required to conduct 4 successive simulated Night shifts (22:00-9:00) and subsequent Day Sleep 1-4 (12:00-18:00). Beginning on the eighth day and continuing for a total of $3 \mathrm{~d}$, participants were again required to be in bed from 0:00 to 7:00 (Recovery Sleep $1-3)$. On the eighth day, they were confined to the living quarters where they were allowed to read a book, watch TV, study, listen to music. From the ninth to tenth day, participants were required to conduct 2 successive simulated Day Shifts (10:00-18:00). On the tenth day, this experiment ended at 18:30 and all the participants left for home. Throughout the experiment, regular balanced meals were provided [Day shifts: 7:30-8:00, 12:00-13:00, 18:30-19:30; Night shifts: 10:00-10:30, 18:30-19:30].

During each shift, participants completed a task (30 $\mathrm{min})$, a computerized test battery (20 min) and break times $(10 \mathrm{~min})$ each hour. The task involved encoding documents in a computer. The computerized test battery consisted of a visual vigilance test (VVT), and subjective assessment of sleepiness and fatigue on a visual analogue scale. The VVT required the participants to press the key corresponding to the numeral $(2,4,6,8)$ presented in the center of the display as quickly and accurately as possible. The stimulus was presented for $5 \mathrm{~s}$ and the interstimulus interval randomly varied between 1 and $10 \mathrm{~s}$. Response times longer than $5 \mathrm{~s}$ were scored as lapses.

Participants spent their free time reading books, studying, watching TV, chatting, and listening to music in the laboratory. For the entire experimental period, participants were prohibited from taking naps and ingesting caffeine or other stimulants. The experimenters monitored the participants from a separate room throughout the experiment.

Sleep was monitored by a wrist actigraph (Mini Mitter Company, Inc. Bend, OR,USA; Actiwatch $\left.64^{\circledR}\right)$. The reliability and validity of this device was confirmed by previous research ${ }^{11)}$. Sleep data were analyzed by ActiwareSleep ${ }^{\circledR}$ ver. 3.2 software (Mini Mitter Company, Inc. Bend, OR, USA). Epoch length was set at one-minute intervals. The actigraph was secured on the participant's non-dominant wrist. The present study focused on sleep efficiency (SE) as an indicator of sleep recovery, since the SE, calculated as the ratio of total sleep time (TST) to total time in bed (TIB) $\times 100$, is proposed to be closely related to subjective sleep quality ${ }^{12)}$ [TIB, beginning when the participant tried to fall asleep and ending when he got out of bed; TST, the time defined as sleep during TIB]. We defined poor sleep as a SE of $80 \%$ or lower during the recovery sleep periods ${ }^{13)}$.

Table 1 shows the SE per participant in each sleep period. Over the 3 successive nights of recovery sleep, six, 
Table 1. Individual sleep efficiencies for baseline sleep and recovery sleep periods 1 to 3

\begin{tabular}{ccccc}
\hline Participant & Baseline Sleep & Recovery Sleep 1 & Recovery Sleep 2 & Recovery Sleep 3 \\
\hline P-01 & 87.4 & 76.4 & 47.4 & 85.2 \\
P-02 & 97.6 & 96.7 & 96.0 & 96.7 \\
P-03 & 97.1 & 36.4 & 61.0 & 61.4 \\
P-04 & 85.2 & 87.1 & 86.0 & 88.3 \\
P-05 & 88.8 & 89.8 & 92.1 & 92.6 \\
P-06 & 81.4 & 28.8 & 57.9 & 81.4 \\
P-07 & 87.9 & 27.6 & 91.7 & 73.6 \\
P-08 & 85.5 & 81.7 & 91.7 & 82.4 \\
P-09 & 79.8 & 29.5 & 93.1 & 79.8 \\
P-10 & 87.4 & 76.4 & 68.6 & 85.2 \\
\hline
\end{tabular}

Sleep efficiency (the ratio of total sleep time to total time in bed $\times 100$, SE) was evaluated by a wrist actigraphy (Mini Mitter Company, Inc.; Actiwatch64). Shadow blocks represent "poor sleep" defined by SE of 80\% or lower.
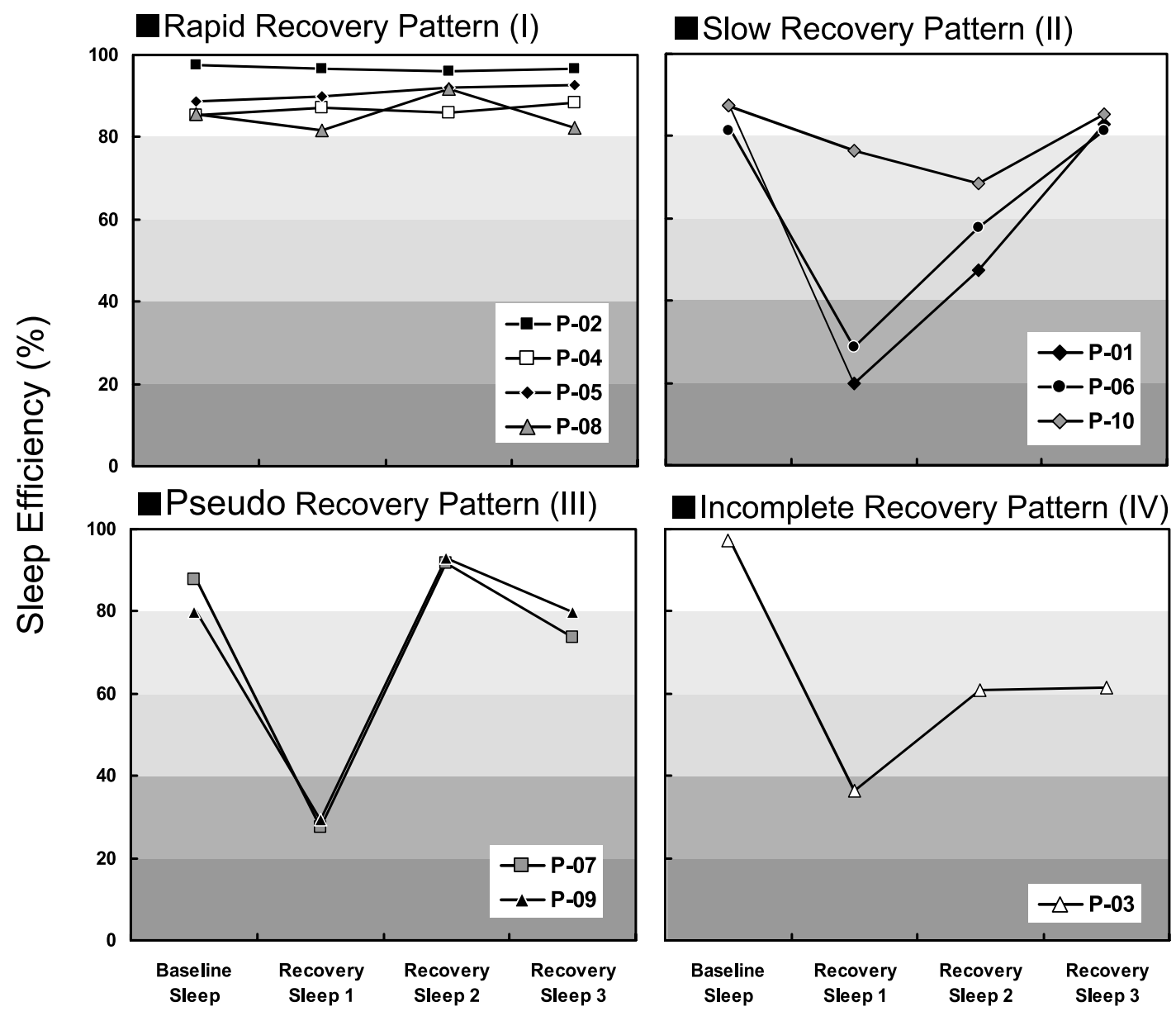

Fig. 1. Four patterns of recovery according to the variation of sleep efficiencies during the recovery periods.

four and three out of the total participants, respectively, showed poor sleep. These data may indicate overall recovery of sleep after the night shifts, but it should be noted that the interindividual differences were considerable.

We categorized the participants into four groups according to the variation of the SE recorded during the Recovery sleep 1, 2 and 3: (I) rapid, (II) slow, (III) pseudo, and (IV) incomplete recovery groups (Fig. 1). The rapid recovery group $(n=4)$ showed immediate return to baseline levels of SEs prior to the night shifts, with high 
levels of SE during the Recovery sleep. The slow recovery group $(n=3)$ did not recover their SEs until Recovery sleep 3. In contrast, the remaining two groups failed to recover even by the third night of recovery sleep. Thus, the pseudo recovery group $(n=2)$ exhibited poor sleep at Recovery sleep 3, despite transient recovery at Recovery sleep 2. The incomplete recovery group $(\mathrm{n}=1$ here $)$ showed consistently poorer sleep (SE of at most 60\%) during the entire recovery periods.

Assuming that sleep habits prior to experiment and/or alertness and performance during the simulated night shifts may be related to the differences in recovery patterns, we focused on the following 8 variables: mean bed time before the experiment, mean wake time before the experiment, variation of bed time before the experiment, variation of wake time before the experiment, morningness-eveningness questionnaire score ${ }^{10)}$, sleep quality during day sleep, sleepiness during night shifts, and visual vigilance test (VVT) lapses during night shifts (see Fig. 2). We then examined the relationship between the recovery patterns and these variables using Spearman's rank correlation coefficient. Significant correlations were observed for mean bed time $\left(\mathrm{r}_{\mathrm{s}}=0.642, p=0.045\right)$ and variation of wake time $\left(\mathrm{r}_{\mathrm{s}}=0.700, p=0.024\right)$ (Fig. 2), Although the $p$ value did not reach a significant level, a moderate correlation coefficient was found between the recovery pattern and chronotype $\left(\mathrm{r}_{\mathrm{s}}=0.572, p=0.084\right)$, possibly due to an apparent U-shaped relationship in which the MEQ scores in both the rapid and incomplete recovery groups tended to be morning type compared to the other groups. There were no significant results for the other variables. Moreover, we examined the possibility that any differences between mean sleep time before the experiment and Baseline Sleep would affect the recovery patterns by Spearman's rank correlation coefficient, but no significant correlation was found $\left(r_{\mathrm{s}}=-0.17, p=0.623\right)$.

The primary finding of the present study was that the individual patterns of sleep recovery following the four night shifts could be categorized into four (i.e., rapid, slow, pseudo, and incomplete) possible types. This is interesting since the fact that the participants were selected according to the standard inclusion and exclusion criteria that are common to most sleep research. Given the paucity of evidence for the individual differences in recovery of sleep after the successive night shifts, the present data may provide novel insights into the recovery process from sleep-deprived circumstances such as night shifts and extended operations.

The present study found that the recovery patterns were significantly related to sleep habits prior to the experiment, more specifically, mean bed time and mean wake time variability before experiment, but not to alertness and performance during the simulated night shifts. Evidence about factors relevant to the recovery process is very limited. Some findings report systematic predictors for the individual differences during sleep deprivation, but no data has been presented for the factors to predict individual variability to recovery from sleep deprivation ${ }^{7}$. The latest study found that the amount of night sleep obtained prior to sleep restriction had not only a favorable influence on performance and alertness during the restriction period, but also facilitated recovery in the postrestriction period ${ }^{14)}$. However, that study did not manipulate the timing of sleep before the sleep restriction. Further investigation is thus necessary to examine how the recovery process is varied by the sleep timing, in particular bed time and wake-time regularity, before a period of sleep loss.

The results of the present study did not show a significant relationship between the MEQ score and the recovery patterns. It is thus likely that the sleep recovery might be affected more predominantly by other factors (ex. sleep length or variation of sleep time prior to the experiment). Indeed, a previous study reported that MEQ scores of individuals were not equally well described by their clock gene PERIOD $3^{15}$.

Among the four types of sleep recovery that were categorized here, the rapid and incomplete recovery groups were seen as two extremes. The former was able to return to the baseline SE immediately following the night shifts, whereas the latter showed no recovery even by the third night following the night shifts. These individual patterns have not been described in the previous research where averaged data have been used ${ }^{5,15,16)}$. Although the correlations were not statistically significant, the incompletely recovered participant was found to yield higher performance (fewer VVT lapses) and alertness (lower subjective sleepiness) during the night shifts and better sleep quality during day sleeps than the other participants (Fig. 2). Probably, this participant might have had to pay a huge "recovery cost" to return from night shifts with a greater level of adaptation to a day-oriented schedule.

Several limitations of this study warrant mention. First of all, we could not examine the underlying mechanism of individual differences in recovery to successive night shifts. Secondly, it is possible that our classification for sleep recovery patterns might have been arbitrary because the results may be different with lager sample sizes. However, it was hard to find helpful information about how to classify the individual patterns of sleep recovery following sleep deprivation or night shifts. Thirdly, it is possible that recovery patterns of sleep might be changed with respect to the sorts of work load (ex. physical or mental load), age, sex, and lifestyle. Finally, the small sample size makes our findings tentative and less robust. Further investigations with larger samples are necessary. 

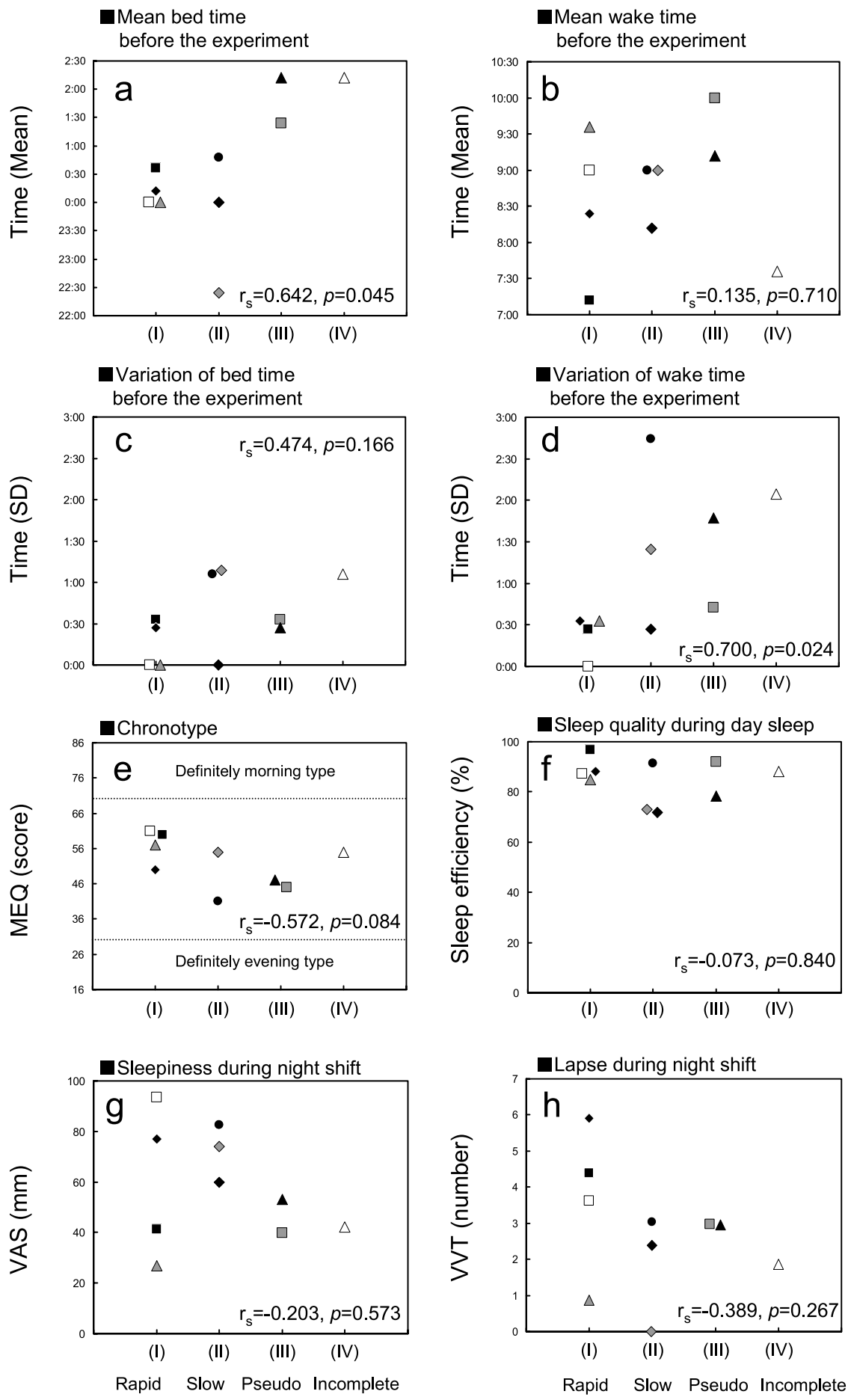

$$
\begin{aligned}
& \text { (I) } \square \mathrm{P}-02 \quad \square \mathrm{P}-04 \bullet \mathrm{P}-05 \triangle \mathrm{P}-08 \\
& \text { (II) } \diamond \mathrm{P}-01 \bullet \mathrm{P}-06 \diamond \mathrm{P}-10 \\
& \text { (III) } \square \mathrm{P}-07 \quad \Delta \mathrm{P}-09 \\
& \text { (IV) } \triangle \mathrm{P}-03
\end{aligned}
$$

Fig. 2. Relationship between four recovery patterns and eight variables.

(a) Mean bed time during one week prior to the experiment by self reported sleep log. (b) Mean wake time during one week prior to the experiment by self reported sleep log. (c) Standard deviation of bed time during one week prior to the experiment by self reported sleep log. (d) Standard deviation of wake time during one week prior to the experiment by self reported sleep log. (e) Morningness-eveningness questionnaire (MEQ) score by Horne and Östberg. (f) Mean percentage of sleep efficiencies during four day sleeps after night shifts evaluated by a wrist actigraph (Mini Mitter Company, Inc.; Actiwatch64 ${ }^{\circledR}$ ). (g) Mean value of visual analogue scale for sleepiness during four successive night shifts. (h) Mean number of lapses (> $5 \mathrm{~s}$ ) for visual vigilance test during four successive night shifts. All correlations were tested by Spearman's rank correlation coefficient. 
In conclusion, the present study showed that recovery patterns of sleep following simulated, four successive night shifts differed considerably among individuals, implying four possible types of recovery. The results also suggest that individual differences in recovery from night shifts may be related more closely to sleep habits prior to the experiment, rather than to performance and alertness during the night shifts.

\section{Acknowledgement}

This study was supported by a Grant-in-Aid for Occupational Medicine Research from JAPAN POST (2005). This study was presented at the SLEEP 2007 21st Annual Meeting of the Associated Professional Sleep Societies (Minnesota, USA, 2007) and the 18th JapanChina-Korea Joint Conference on Occupational Health (Nagoya, Japan, 2007).

\section{References}

1) Kogi K (1982) Sleep problems in night and shift work. J Hum Ergol 11, 217-31.

2) Folkard S, Tucker P (2003) Shift work, safety and productivity. Occup Med (Lond) 53, 95-101.

3) Shift Work Committee, Japan Association of Industrial Health (1979) Opinion on night work and shift work. J Sci Lab 55, 1-36.

4) Knauth P, Rutenfranz J (1982) Development of criteria for the design of shiftwork system. J Hum Ergol 11, 337-67.

5) Bjorvatn B, Stangenes K, Oyane N, Forberg K, Lowden A, Holsten F, Åkerstedt T (2006) Subjective and objective measures of adaptation and readaptation to night work on an oil rig in the North Sea. Sleep 29, 821-9.

6) Lamond N, Dorrian J, Burgess H, Holmes A, Roach G, McCulloch K, Fletcher A, Dawson D (2004) Adaptation of performance during a week of simulated night work. Ergonomics 47, 154-65.

7) Van Dongen HP, Baynard MD, Maislin G, Dinges DF (2004) Systematic interindividual differences in neurobehavioral impairment from sleep loss: evidence of trait-like differential vulnerability. Sleep 27, 423-33.

8) Viola AU, Archer SN, James LM, Groeger JA, Lo JC, Skene DJ, von Schantz M, Dijk DJ (2007) PER3 polymorphism predicts sleep structure and waking performance. Curr Biol 17, 613-8.

9) Bjorvatn B, Pallesen S (2009) A practical approach to circadian rhythm sleep disorders. Sleep Med Rev 13, 47-60.

10) Horne JA, Östberg O (1976) A self-assessment questionnaire to determine morningness-eveningness in human circadian rhythms. Int J Chronobiol 4, 97-110.

11) Tonetti L, Pasquini F, Fabbri M, Belluzzi M, Natale V (2008) Comparison of two different actigraphs with polysomnography in healthy young subjects. Chronobiol Int 25, 145-53.

12) Åkerstedt T, Hume K, Minors D, Waterhouse J (1994) The meaning of good sleep: a longitudinal study of polysomnography and subjective sleep quality. J Sleep Res 3, 152-8.

13) Lichstein KL, Durrence HH, Taylor DJ, Bush AJ, Riedel BW (2003) Quantitative criteria for insomnia. Behav Res Ther 41, 427-45.

14) Rupp TL, Wesensten NJ, Bliese PD, Balkin TJ (2009) Banking sleep: realization of benefits during subsequent sleep restriction and recovery. Sleep 32, 311-21.

15) Groeger JA, Viola AU, Lo JC, von Schantz M, Archer SN, Dijk DJ (2008) Early morning executive functioning during sleep deprivation is compromised by a PERIOD3 polymorphism. Sleep 31, 1159-67.

16) Belenky G, Wesensten NJ, Thorne DR, Thomas ML, Sing HC, Redmond DP, Russo MB, Balkin TJ (2003) Patterns of performance degradation and restoration during sleep restriction and subsequent recovery: a sleep dose-response study. J Sleep Res 12, 1-12. 